\title{
Estimates of Efficiency and Control Methods of Communication Network Functioning
}

\author{
Mirzaeva Malika Bakhadirovna ${ }^{1}$,SobirovMuzaffar Azatovich ${ }^{2}$ \\ ${ }^{1}$ Tashkent University of Information Technologies named after Muhammad al-Khwarizmi, Uzbekistan, \\ malikamirzaeva01@gmail.com \\ ${ }^{2}$ Tashkent University of Information Technologies named after Muhammad al-Khwarizmi, Uzbekistan, \\ muzaffar-sobirov@mail.ru
}

\begin{abstract}
This article analyzes the criterion for assessing the efficiency of the studied communication network and a systematic approach to assessing the effectiveness of networks. A method for evaluating the effectiveness of the functioning of a communication network is proposed, including the developed models for assessing the effectiveness of the functioning of a communication network and a program for calculating a generalized criterion. The results of experiments carried out at different values of the input flow intensity are presented. A methodology for managing the functioning of the communication network, which allows providing the necessary value of the efficiency of the network functioning are developed.
\end{abstract}

Key words: indicator, communication network, backup channels, flow intensity, signal frequency.

\section{INTRODUCTION}

The usual approach to solving these problems is to compare networks by individual parameters. However, this approach can lead to satisfactory results only in some frequent cases, for example, when two networks are compared and one of them is better in all parameters (or in the vast majority of parameters) than the other. In the general case, when several networks are compared, the best in some parameters is the worst in others, this approach does not allow achieving the goal of choosing one best option.

In this regard, there is a need for a generalized criterion for assessing the network in the form of a single numerical value that fully characterizes the systemic consideration of the communication network, which is expressed in the system approach. The specificity of the systematic approach is manifested primarily in the fact that the assessment is carried out not according to individual criteria, but in general according to a set of criteria.

\section{A SYSTEMATIC APPROACH TO ASSESSING THE EFFECTIVENESS OF COMMUNICATION NETWORKS}

Thus, the essence of systemic income for assessing the effectiveness of communication networks consists in constructing an assessment of efficiency in a form that would cover all the main parameters and reflect the ability of the network to solve functional problems.

When constructing a generalized criterion for assessing efficiency, functional tasks should be understood as tasks to ensure individual qualities of the system. The presence of separate functional tasks allows you to select from all parameters of the system $\left(\alpha_{1}, \alpha_{2}, \ldots . \alpha_{n}\right)$ parameter group $\left(\alpha_{1}, \alpha_{2}, \ldots . \alpha_{k}\right)$, reflecting the quality of performance of the relevant functional tasks. Each functional task can be associated with a certain objective function, which quantitatively characterizes the degree of achievement of the set goals [1]. In general, the objective function for the network is an arbitrary dependence on the parameters affecting its performance. An estimate based on the weighted summation of the system parameters is very simple and at the same time flexible

$$
\gamma=\sum_{n=1}^{N} c_{n} * \alpha_{n},
$$

where: $N$ - number of parameters taken into account;

$c_{n}$ - weighting factors;

$\alpha_{n}$ - parameters.

Only the requirement for the objectivity of the assessment needs special reservation. Indeed, some degree of subjectivity is inevitable when choosing the weighting factors.

The disadvantages of estimate (1) can also be attributed to the linear form, since nonlinear dependences are able to more subtly reflect the influence of individual parameters. Among the nonlinear estimates proposed recently, the estimate in the form of a postnominal meets the necessary requirement quite fully.

where

$$
\gamma=\prod_{n=1}^{N} \alpha_{n}^{c_{n}}
$$

$\alpha_{n}$ - system parameters;

$c_{n}$ - weighting factors.

However, the direct substitution of the values of the selected criteria for the efficiency of the communication network functioning into formula (2) will not give an idea of the network efficiency in the sense in which we defined it. In this regard, in (2), instead of indicators $\alpha_{n}$ the quantities should be used $R_{j}$, reflecting the degree of compliance of a particular criterion with the required values. Such quantities are:

$$
R_{1}=T_{g}^{*} / T_{g} ; \quad R_{1}=H / H^{*} ; \quad R_{3}=1-\frac{\left|P_{\text {error }}^{*}-P_{\text {error }}\right|}{P_{\text {error }}^{*}+P_{\text {error }}}
$$

where $H, H^{*}$ - respectively the calculated and required indicator of the reliability of the communication network; $T_{g}, T_{g}^{*}$ - respectively, the calculated and required values of 
the time for delivering messages over the communication network;

$P_{\text {error }}, P_{\text {error }}^{*}$ - respectively, the calculated and required probability of error transmission of messages over the communication network.

The quantity $R_{1}$ can take any value. The values $R_{2}$ do not exceed one, since the functional reliability indicator is focused on the maximum possible load. Expression for $R_{3}$ is chosen so that it does not exceed one, i.e. are ineffective as values $P_{\text {error }}<P_{\text {error }}^{*}$, so and $P_{\text {error }}>P_{\text {error }}^{*}$.

The latter is due to the fact that at $P_{\text {error }}<P_{\text {error }}^{*}$ either excessive redundancy of the code is used, leading to an increase in the delay of messages over the network [2], or a spare frequency, or transmission with excessive signal power is carried out.

Based on the above, as a generalized criterion for the effectiveness of the functioning of the studied communication network, we propose

$R_{\text {common }}=R_{1}^{c_{1}} * R_{2}^{c_{2}} * R_{3}^{c_{3}}$

Thus, a target function (generalized criterion) has been synthesized, which makes it possible to assess the effectiveness of the functioning of the communications network under study according to the selected criteria; in other words, to determine the degree of compliance of the communication network functioning with the requirements set by the users.

The practice of the expert survey has shown that its success significantly depends on the number of initial parameters: the more parameters are to be assessed, the greater the set of parameters experts assign the same importance. In other words, with an increase in the number of parameters, experts become more and more indifferent in their judgments. Since, in the general case, the number of initial parameters cannot be reduced without prejudice to the completeness of the network description, it is advisable to introduce a generalized criterion.

\section{METHOD FOR ASSESSING THE EFFECTIVENESS OF THE COMMUNICATION NETWORK}

To assess the efficiency of the communication network under consideration according to the generalized criterion (3), a set of programs is proposed (Figure 1), including the developed models for assessing the effectiveness of the communication network and the program for calculating the generalized criterion, which are organized in the form of separate modules. The sequence of their implementation depends on the task at hand.

The complete cycle of the complex operation can be divided into the following stages:

1. Using the simulation model of the considered communication network and the correction algorithm, the maximum message delay on the network routes with ideal reliability is determined.

2. When using the channel model with real reliability, the maximum message delay on the network routes is estimated taking into account the flows of failures and restoration of communication channels.

3. When using the obtained values of the optimal plan for the distribution of the input flow along the routes of the network with real reliability and the model of the channel with interference using the simulation model of the communication network, the reliability of the messages transmitted over the network is assessed by the frequency of distorted messages.

4. Based on the found values of the efficiency criteria, a generalized criterion of the efficiency of the functioning of the investigated communication network is calculated.

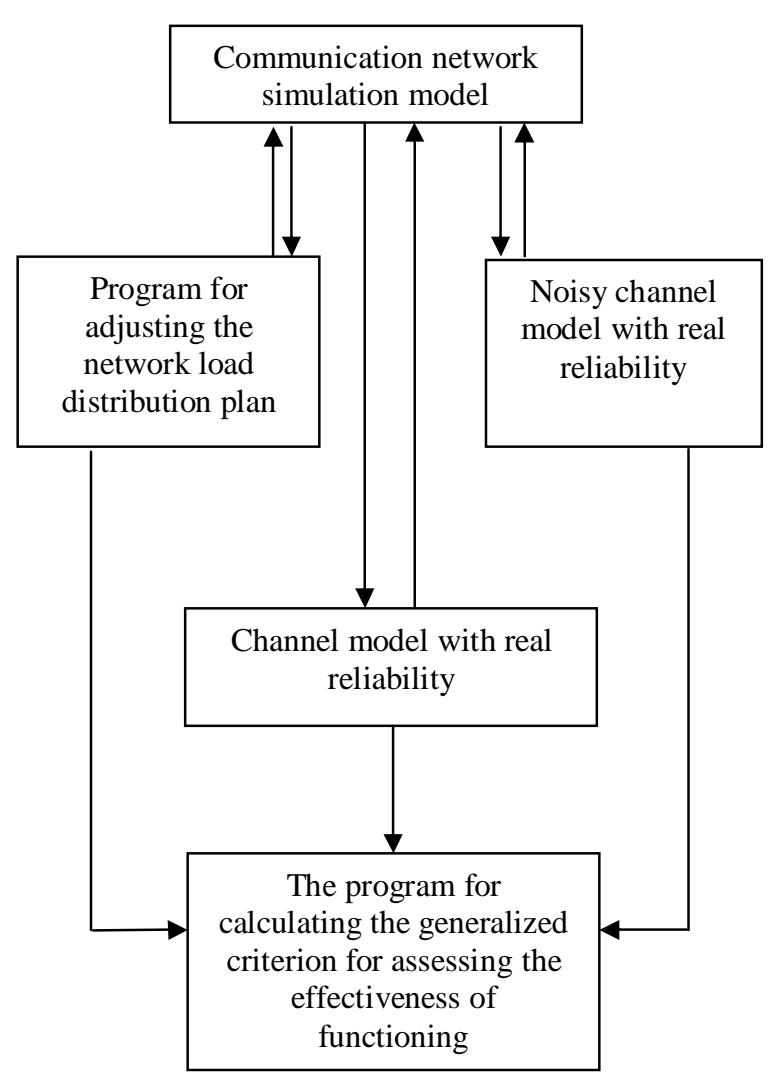

Figure 1: Complex of programs for assessing the effectiveness of the communicationnetwork

This question cannot be answered unequivocally. The point is that, although the selected criteria are interrelated, the compensation of the "bad" value of any parameter with the "good" values of others may not provide the required level of efficiency [3-4]. For example, consider the case when the lead time is greater than the required value, and the error probability is less than the required one. Then by reducing the redundancy used when we can reduce the transmission time of messages. However, by reducing the confidence to the lower threshold $\left(R_{3}=1\right)$ this may not reach the required time for delivering messages over the network. Mutual compensation by the criterion can be carried out after appropriate control of the communication network. If full compensation is not achieved, then it is necessary to use additional (spare) network resources or limit the incoming flow of messages.

Based on the specifics of the communications network under study, which is characterized by the presence of severe restrictions and problems associated with compensation, for the case when at least one of the parameters $R_{j}, j=\overline{1,3}$, does not meet the requirements, we suggest evaluating the efficiency of the considered communication network according to the criterion

$R_{\text {common }}=\min \left\{R_{1}, R_{2}, R_{3}\right\}$ 
Thus, to assess the efficiency of the functioning of the considered multi-level hierarchical communication network, including different types of communication channels, a generalized criterion is proposed:

$$
R_{\text {common }}=\left\{\begin{array}{c}
R_{1}^{c_{1}} * R_{2}^{c_{2}} * R_{3}^{c_{3}}, \text { if } \forall_{j}\left(R_{j} \geq 1\right) \\
\min \left\{R_{1}^{c_{1}} * R_{2}^{c_{2}} * R_{3}^{c_{3}}\right\}, \text { if } \exists_{j}\left(R_{j}<1\right)
\end{array}\right\}
$$

Table 1 shows the results of experiments carried out at different values of the input flow intensity $\left(\lambda_{\text {input }}=\right.$ $0,5 \ldots 10$ message/ $s$ )messages of the same type with a length of 500 bits and unchanged characteristics of channels and communication nodes [5]. In this case, the unit values of the weight coefficients are $\operatorname{taken}\left(C_{j}, j=\right.$ $\overline{1,3})$.

Table 1: Results of experiments carried out at different values of the input flow intensity

\begin{tabular}{|l|l|l|l|l|}
\hline$\lambda_{\text {input }}$ & $T_{g}^{j} \max , s$ & $\mathrm{H}$ & $\rho_{\text {error }}$ & $R_{\text {common }}$ \\
\hline 0,5 & 3,25 & 0,85 & $0,26^{*} 10^{-2}$ & 2,45 \\
\hline 1,0 & 4,39 & 0,87 & $0,26^{*} 10^{-2}$ & 1,85 \\
\hline 2,0 & 4,71 & 0,86 & $0,24^{*} 10^{-2}$ & 1,83 \\
\hline 2,5 & 5,27 & 0,88 & $0,24^{*} 10^{-2}$ & 1,39 \\
\hline 3,5 & 6,48 & 0,87 & $0,27^{*} 10^{-2}$ & 1,16 \\
\hline 5,0 & 7,76 & 0,81 & $0,24^{*} 10^{-2}$ & 0,84 \\
\hline 6,5 & 9,34 & 0,79 & $0,25^{*} 10^{-2}$ & 0,71 \\
\hline 8,0 & 12,02 & 0,74 & $0,26^{*} 10^{-2}$ & 0,54 \\
\hline 10,0 & 13,78 & 0,73 & $0,27^{*} 10^{-2}$ & 0,42 \\
\hline
\end{tabular}

The values of the generalized criterion given in Table 1 are calculated with the following required values of the efficiency criteria of the considered communication network: $T_{g}^{j}=6,5 s ; \bar{H} \mathrm{H}^{*}=0,85 ; \rho_{\text {error }}^{*}=0,28 * 10-2$.

Table 1 shows that the values of the reliability indicators of the communication network and the average error probability of messages transmitted over the network vary randomly around some average value. This is due to the fact that with constant characteristics of channels and nodes of the communication network (probability of failure, the law of distribution of noise amplitudes, etc.), these criteria do not depend on the value of the input stream. Therefore, in this case, by the value of the generalized criterion $\left(R_{\text {common }}\right)$ only the value of the maximum delay of messages on the network affects. With an increase in the intensity of the input stream, the maximum message delay on the network increases, in order to lead to a decrease in the generalized criterion for evaluating the communication efficiency. Atintensityvalue $\lambda_{\text {input }}=0,5$ message $/ \mathrm{s}$ it gets the maximum delay, equal $7,76 \mathrm{~s}$, what corresponds $R_{\text {common }}=0,84$.

Toachievetherequiredparametervalue $R_{\text {common }}$ under $\lambda_{\text {input }}=0,5$ message $/$ sit is necessary to carry out some control action (for example, limiting the input flow, changing the routing algorithm, introducing redundant elements, etc.) to normalize the efficiency of the network.

\section{METHODOLOGY FOR MANAGING THE OPERATION OF THE COMMUNICATION NETWORK}

In order to develop some control effect on the communication network, allowing to ensure the required level of its functioning, it is necessary from the set $\mathcal{W}_{0}$ permissible impacts to develop a subset of possible impacts: $\left\{w_{i}\right\} \subset \mathcal{W}_{0}$.

The adoption of the final decision on control, as a rule, is based not only on the value of the generalized assessment of the state of the communication network, but also on the account of some additional set of information about the control object and the specifics of the current situation [6]. Accounting for this information is usually difficult to formalize and is carried out by experts who have extensive experience in managing this communication network. Therefore, it is impossible to identify the generalized assessment of the effectiveness of the communication network with the final criterion for making a decision on management, since the assessment only prepares, justifies decisions in quantitative terms. Foranexpertfromthemultitude $\left\{w_{i}\right\}$ could easily select the best control action in accordance with additional criteria, it is necessary that all possible actions $w_{i},(i=1,2, \ldots)$ were equal. So,thechallengeistofindsuchvalues $\left\{\left|w_{i}\right|\right\}_{\text {which }}$ lead to the same value of the generalized criterion $R_{\text {common }}=$ 1 .

Due to the fact that the selected criteria for assessing the efficiency of the communication network functioning are interconnected, when choosing the necessary control actions, the question arises: in what sequence to influence the parameters in order to avoid re-examining some of them after changing the values of others? Let's consider these parameters (evaluation criteria): reliability, delivery time and reliability.

FunctionalreliabilityindicatorHdependsonnetworkstructure $Z$, failure rates $\left\{\delta_{i}\right\}$ and recovery $\left\{v_{i}\right\}$ network elements, load balancing plan and number of retransmissions. Timetobring $T_{g}$ the message depends on the same characteristics, as well as on the size of the incoming stream $\lambda_{\text {input }}$. The reliability depends on factors such as the level of interference, the method of encoding, the frequency and power of the transmitted signal, etc.

Control actions aimed at increasing functional reliability Hcommunication networks also lead to a decrease in the time of $T_{g}$. However, by reducing the delivery time, we cannot always achieve an increase in network reliability, since a decrease in message delays over the network can be achieved by limiting the flow - a control action that does not affect the valueH. Hence it follows that when choosing control actions, it is advisable to consider firstH, then $T_{g}$.

Therelationshipbetween $\rho_{\text {error }}$ andHis also one-sided. Control actions aimed at increasing reliability affect the functional reliability of the network, and not vice versa. For example, an increase in the number of retransmissions decreases the values of $\mathrm{H}$, since it leads to a decrease in the real network throughput. At the same time, none of the control actions allowing to increaseH, do not affect the accuracy $\rho_{\text {error }}$. Therefore, it is advisable to consider first $\rho_{\text {error }}$, thenH.

Therefore, when choosing control actions aimed at increasing the efficiency of the communication network functioning, the selected efficiency criteria should be considered in the following order: reliability, reliability, delivery time [7]. Moreover, it is enough to consider each of the criteria once, without returning to it after changing the values of the others. 
Thequantities $R_{2}$ and $R_{3}$ shouldbereducedtooneandinthosecas eswhen $R_{2}>1$ and $R_{3}>1$, since in the first case we have unnecessary costs, and in the second - possible losses due to a decrease inHandincrease $T_{g}$.

Suppose that in the result of the assessment of the effectiveness of the operation of the considered communication network, it obtains $R_{\text {common }}<1$, which corresponds to the ineffective level of operation and the need for network management.

Based on the above considerations, first of all we need to give $R$ to the norm. Suppose we have at our disposal the following sets of control actions that provide the required level of confidence: changing the code, signal frequency, signal strength, code and frequency, power and code, frequency and power, code, frequency and power. Of these, only those are considered that provide the required value $\rho_{\text {error }}$. From the subset of the considered options, leading to the same value $R_{3}=1$, one is selected by an expert according to non-formalized criteria.

Further, havingachievedthat $R_{3}=1$, as a result of assessing the functioning of the network, we can get again $R_{\text {общ }}<1$. Nowweneedtoprovide $R_{2}=1$.Suppose there are the following control actions aimed at normalizingH: increased intensity and recovery;

connection of backup channels;

connection of redundant nodes;

increasing the intensity of restoration and connecting backup channels;

connectionofbackupchannelsandnodes.

Thesizesofthecontrolactionsarecalculatedsothat $R_{2}=1$, and among them the expert chooses the best impact depending on additional criteria that are difficult to formalize. When $R_{2} \geqslant 1$, asituation, where $R_{2}<1$, is impossible, since the adopted reliability indicator is focused on the timely delivery of the maximum possible flow. In other words, as a result of the conducted network management, we achieve an assessment $R_{\text {error }} \geqslant 1$, which corresponds to the required level of communication network functioning.

Thus, the essence of the developed methodology for effective management of the operation of the investigated communication network is as follows. The sizes of the control actions are calculated so that $R_{\text {общ }}=1$. Among

Table 2: Results of experiments carried out at different values of the recovery intensity $\delta_{\text {recovery }}$ of data transmission channels them, the expert chooses the best impact according to additional non-formalized (difficult to formalize) criteria.

If $R_{\text {common }}$ cannot be brought to unity, then for all options (or for those selected for research by an expert) the flow is limited in such a way that in each option under consideration there is $R_{\text {common }}=1$.

In this case, the values $\Delta z_{i}-$ flow restrictions. Sinceinthiscaseallcontroloptionsareequivalentby $R_{\text {common }}$, then the expert only has to compare them in accordance with additional non-formalized criteria.

Let us give an example that illustrates the essence of the methodology for managing the operation of a communication network. The experiments were carried out for a priority-free message flow with an arrival rate of 10 messages/s. Let the following admissible values of the network characteristics be given:

$P^{*}=0,25 * 10^{-2}$;

$$
\mathrm{H}^{*}=0,8 ; T_{g}^{*}=7 \mathrm{~s} .
$$

As a result of assessing the current state of the considered communication network using the developed simulation models, we obtained the following values of the network characteristics:

$$
\begin{aligned}
& \rho_{\text {error }}=0,24 * 10^{-2} ; \mathrm{H}=0,67 ; T_{g}=12 \mathrm{~s} . \\
& \text { or } \\
& R_{1}=0,58 ; R_{2}=0,83 ; R_{3}=1,00 ; R_{\text {common }}=0,48 .
\end{aligned}
$$

which corresponds to an ineffective level of functioning. Therefore, it is necessary to control the operation of the communication network.

Accordingtotheproposedmethod, $\quad$ since $R_{3}=1$ and $R_{\text {common }}<1$, it needs to bring $R_{2}$ to the required level. Moreover, providing $R_{2}=1$ different control actions, it is possible to make a choice of the best of equivalent actions according to additional difficult to formalize criteria [8]. For simplicity, we will consider two control actions according to additional criteria that are difficult to formalize. For simplicity, we will consider two control actions: changing the intensity of restoration of network elements and connecting backup channels.

Table 2 shows the results of experiments carried out at different values of the recovery $\operatorname{rate} \delta_{\text {recovery }}$ data transmission channels.

\begin{tabular}{|l|l|l|l|l|l|l|l|l|}
\hline \multicolumn{1}{|c|}{$\lambda_{\text {input }}$} & $T_{g}^{j} \max , s$ & & \multicolumn{1}{|c|}{$\rho_{\text {error }}$} & $R_{1}$ & $R_{2}$ & $R_{3}$ & $R_{\text {common }}$ \\
& & & & & & & \\
\hline 0,010 & 14,1 & 0,6 & $0,25^{*} 10^{-2}$ & 0,5 & 0,75 & 1,00 & 0,38 \\
\hline 0,015 & 13,4 & 0,63 & $0,25^{*} 10^{-2}$ & 0,52 & 0,78 & 1,00 & 0,41 \\
\hline 0,020 & 12,7 & 0,66 & $0,25^{*} 10^{-2}$ & 0,55 & 0,82 & 1,00 & 0,45 \\
\hline 0,025 & 12,1 & 0,67 & $0,25^{*} 10^{-2}$ & 0,58 & 0,83 & 1,00 & 0,48 \\
\hline 0,030 & 10,3 & 0,71 & $0,24^{*} 10^{-2}$ & 0,67 & 0,88 & 1,04 & 0,61 \\
\hline 0,035 & 8,3 & 0,76 & $0,25^{*} 10^{-2}$ & 0,84 & 0,95 & 1,00 & 0,80 \\
\hline 0,040 & 7,6 & 0,79 & $0,25^{*} 10^{-2}$ & 0,92 & 0,98 & 1,00 & 0,90 \\
\hline 0,045 & 6,4 & 0,83 & $0,24^{*} 10^{-2}$ & 1,09 & 1,04 & 1,04 & 1,18 \\
\hline 0,050 & 5,8 & 0,89 & $0,25^{*} 10^{-2}$ & 1,21 & 1,11 & 1,00 & 1,34 \\
\hline
\end{tabular}

Table 2 showsthatinordertoobtain $R_{2}=1$, it is necessary to increase the intensity of restoration of data transmission channels of the considered communication network to $\delta_{\text {recovery }}=0,042$. 
Now let's consider the control action, which consists in connecting backup communication channels.

Let us assume that it has at our disposal three backup channels, which belong to the first route, which is the shortest and busiest. Table 3 shows the results of experiments carried out on the communication network

Table 3: Results of experiments carried out at different values of the recovery intensity $\delta_{\text {recovery }}$ of backup channels when connecting channels.

Table 3 showsthatinordertoachieve $R_{2}=1$, it is necessary to connect two backup channels to the main communication route.

\begin{tabular}{|l|l|l|l|l|l|l|l|}
\hline $\begin{array}{l}\text { Number of backup } \\
\text { channels }\end{array}$ & $T_{g}^{j} \max , s$ & $H$ & $\rho_{\text {error }}$ & $R_{1}$ & $R_{2}$ & $R_{3}$ & $R_{\text {common }}$ \\
\hline 1 & 7,7 & 0,78 & $0,25^{*} 10^{-2}$ & 0,91 & 0,98 & 1,00 & 0,89 \\
\hline 2 & 6,8 & 0,82 & $0,25^{*} 10^{-2}$ & 1,02 & 1,03 & 1,00 & 1,05 \\
\hline 3 & 6,1 & 0,85 & $0,25^{*} 10^{-2}$ & 1,14 & 1,06 & 1,00 & 1,21 \\
\hline
\end{tabular}

Nowknowingthemeanings $\left\{\left|w_{i}\right|\right\}$,

whichleadtothesamevalues $R_{2}$, IIt is enough for an expert to take into account the influence of these influences on additional non-formalized criteria for the efficiency of the network functioning and choose the best of the control actions: either increase the intensity of restoration of communication channels to $\delta_{\text {recovery }}=0,042$, or connect backup channels.

Suppose that we cannot achieve with the control actions at our disposal $R_{2}=1$, i.e., for example, it can increase the intensity of restoration of communication channels only up to $\delta_{\text {recovery }}=0,03$. And in this case, according to the methodology, for each option, such a limitation of the input flow should be implemented $\left(\lambda_{\text {input }}=10\right.$ message $\left./ \mathrm{s}\right), \quad$ to $\quad$ achieve $R_{\text {common }}=1$. So, it was experimentally found that in order to achieve the required level of operation of the communication network with the intensity of restoration of its channels $\delta_{\text {recovery }}=0,03$, it is necessary to limit the input stream to $\lambda_{\text {input }}=7,1$ message $/ \mathrm{s}$, and when connected one backup channel up to $\lambda_{\text {input }}=$ 8,6 message/s. In both cases, we achieve that $R_{\text {common }}=1$. Now it remains for the expert to compare the options in accordance with additional criteria and make a decision on the choice of control action.

Thus, only as a result of the creation of a man-machine control system, it is possible to achieve the effectiveness of the functioning of a complex technical system, which is a communication network, the state of which is characterized by both formalized and non-formalized criteria.

\section{CONCLUSION}

In conclusion, it should be noted that when analyzing the criterion for assessing the efficiency of the functioning of the communication network under study, which is characterized by the presence of severe restrictions on its probabilistic-temporal characteristics, a method for effective control of the communication network was developed, which consists in finding a certain set of alternative control actions, each of which provides the necessary value of the efficiency of functioning networks. The choice of the best of these equivalent control actions is carried out by an expert in accordance with additional non-formalized criteria. With a multi-priority message flow, the selection of control actions is carried out separately for each of the priorities, starting with the highest. It is shown that in order to select the best control actions, it is quite acceptable to assess the efficiency of the communication network by the value of the product of the selected criteria.

\section{REFERENCES}

1. Ahmed, W., Hasan, O., Tahar, S.: Formalization of reliability block diagrams in higher-order logic. $\mathrm{J}$ Appl Logic 18, 19-41 (2016). https://doi.org/10.1016/j.jal.2016.05.007

2. GenadiyZhyrov, SerhiiLienkov, YuriiHusak, HennadiiBanzak, Igor Tolok. Analysis of problem optimization of parameters maintenance process according to state with constant periodicity of control. International Journal of Emerging Trends in Engineering Research. Volume 8. No. 6, June 2020. -PP.2606-2611 https://doi.org/10.30534/ijeter/2020/63862020

3. Netes V.A. Reliability of communication networks during the transition to NGN. M: Vestnik of communication No.-. 2007.

4. Ushakov I.A. Reference: reliability of technical systems. -M: Radio and communications, 1985. -497 pp.

5. Lubov Berkman, Oleg Barabash, Olga Tkachenko, AndriMusienko,OleksandLaptiev, Ivanna Salanda.

The Intelligent Control System for infocommunication networks. International Journal of Emerging Trends in Engineering Research. Volume 8. No. 5, May 2020, -PP.1920-1925 https://doi.org/10.30534/ijeter/2020/73852020

6. Zeliger $\mathrm{NB}$, and others. Designing networks and transmission systems of discrete messages. -M.: radio and communication, 1984. -214 pp.

7. Emelyanov G.A., Schwartsman V.O. Transmission of discrete information. $-\mathrm{M}$.: radio and communications, 1982. $-240 \mathrm{pp}$.

8. Popkov G.V., Levakov A.K. The tasks of optimizing the structural reliability and survivability of communication networks in emergency situations. Vestnik of the Buryat State University. 2013. 